\title{
Instability analysis for a centrifugal pump with straight inlet pipe using partially averaged Navier-Stokes model
}

Proc IMechE Part A:

$J$ Power and Energy

$0(0) 1-16$

(C) IMechE 2020

Article reuse guidelines:

sagepub.com/journals-permissions

DOI: I0.1 I77/09576509209|9540

journals.sagepub.com/home/pia

(9SAGE

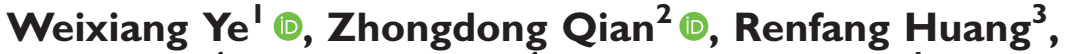 \\ Xiaojun $\mathrm{Li}^{4}{ }^{\circ}$, Zuchao Zhu ${ }^{4}(\mathbb{D}$ and Xianwu Luo'
}

\begin{abstract}
The current study numerically investigates the flow instability under several part-load conditions in a centrifugal pump with a straight inlet pipe to explore the underlying relationship between a positive slope phenomenon and internal flow using a partially averaged Navier-Stokes model. The model was validated by comparing the hydraulic performance and averaged flow in the impeller between the numerical results and experimental data of a tested pump. The internal flows in pumps have been intensively investigated based on Batchelor vortex family, Rayleigh-Taylor criterion, entropy generation rate, and energy equation to analyze the flow instability from different aspects. The simulation results using partially averaged Navier-Stokes model are acceptable due to the good agreement with the experimental data for the tested pump. No matter the geometry of the inlet pipe, the pre-swirling flows in the inlet pipe are in the convective instability region. Under the part-load condition of $\phi=0.5 \phi_{\text {bep }}$, the axial vorticity coefficient is affected by the geometry of the inlet pipe. However, under the part-load condition with rotating stall, e.g. $\phi=0.78 \phi_{\text {bep }}$, the flow in the inlet pipe is affected by the unstable flow in the pump impeller. For the pump with a straight inlet pipe, the vortex inside the blade-to-blade passage is in a stable state according to Rayleigh-Taylor criterion under the condition of $\phi=0.5 \phi_{\text {bep. }}$ However, the vortex in the blade-to-blade passage is in an unstable state due to centrifugal instability under those operation conditions with rotating stall cells in the impeller, and the dominant oscillations are dependent on the propagation of rotating stall cells. Finally, head loss analysis based on energy equations elucidates that turbulent kinetic energy production term is predominant in the head loss in pump impeller. The present results are helpful for better understanding of the unstable flows and positive slope phenomenon for centrifugal pumps.
\end{abstract}

\section{Keywords}

Centrifugal pump, inlet pipe, positive slope phenomenon, PANS model

Date received: 18 February 2019; accepted: 19 March 2020

\section{Introduction}

Substantial phenomena associated with flow instability characteristics have been investigated in decades. In some present industrial applications such as the gas turbine combustors, ${ }^{1}$ draft tubes of Francis turbines, ${ }^{2,3}$ guide vanes in pump-turbines, ${ }^{4,5}$ and centrifugal pumps, ${ }^{6,7}$ instability phenomena focused on turbulence swirling flows are quite common in general. It is well known that the flow instability investigation concentrated on revolving fluid flow was initially introduced by Lord Rayleigh ${ }^{8}$ in 1917. However, the complex components of the velocity severely complicate research on the mechanism of swirling flow during a period of time. ${ }^{9}$ Nearly half a century ago, Batchelor ${ }^{10}$ proposed the velocity profiles in the steady trailing line vortices and gave a similarity solution for a swirling wake flow in a trailing vortex far downstream. After several decades' efforts, the instability analysis based on the Batchelor's similarity solution has been widely utilized

\footnotetext{
'State Key Laboratory of Hydroscience and Engineering, Department of Energy and Power Engineering, Tsinghua University, Beijing, China ${ }^{2}$ State Key Laboratory of Water Resources and Hydropower Engineering Science, Wuhan University, Wuhan, China

${ }^{3}$ Key Laboratory for Mechanics in Fluid Solid Coupling System, Institute of Mechanics, Chinese Academy of Sciences, Beijing, China

${ }^{4}$ Key Laboratory of Fluid Transmission Technology of Zhejiang

Province, Zhejiang Sci-Tech University, Hangzhou, China
}

\section{Corresponding author:}

Xianwu Luo, State Key Laboratory of Hydroscience and Engineering, Tsinghua University, Beijing 100084, China.

Email: luoxw@tsinghua.edu.cn 
and even developed. For example, Lessen et al. ${ }^{11}$ believed that the stability of the flow could be characterized by a swirl parameter $q$ and azimuthal wave number $n$. Delbende et al. ${ }^{12}$ pointed out that the absolute and convective instabilities in the Batchelor vortex could be determined by an external flow parameter. Galvan et al. ${ }^{13}$ applied three different vortex equations for representing the inlet velocity profiles of a cone diffuser based on the Batchelor vortex family and presented an analysis of the influence of the swirl number on the behavior of the optimization process successfully. It is identical among these studies that non-dimensional velocity components i.e. radial, azimuthal, and axial velocity components are established from the "Batchlor vortex family." Although the swirling flow downstream a runner or in a draft tube has been extensively investigated for instability analysis, ${ }^{2,3,13}$ there are few studies presenting the turbulence flow upstream the impeller inlet in a centrifugal pump based on the Batchelor vortex family. In addition, the complex vortex structures upstream the impeller complicate the flow structures in the impeller, which turns out to be much difficult to reveal the mechanism of the flow instability. Therefore, the analysis on flow instability upstream the impeller is urgent to be carried out.

A centrifugal pump is a kind of power facility which is applied to various technology fields like aerospace, power plants, agriculture, biomedicine, and some other fields. In the field of fluids engineering, the pump-storage schemes are regarded as the most promising energy storage technology recently, meeting the requirements for different electricity grid loads. ${ }^{14,15}$ Therefore, it is of great significance to guarantee the safety and stability of the units, and much attention has been attracted to the instability phenomena as well as its dynamics mechanisms recently. However, under the part-load conditions of the pumps, the non-equilibrium instantaneous radial forces could also be induced by the blade-tongue interference and non-axisymmetric flow structures. ${ }^{16}$ These would result in many adverse phenomena such as noise and intense vibrations. ${ }^{17}$

In the past, a number of researches ${ }^{17-20}$ have focused on the instabilities within the centrifugal pumps by means of experiment and computational fluid dynamics (CFD). Among those papers, entropy generation defined in Yan et al. ${ }^{17}$ is regarded as a parameter to measure the energy loss in a pump. Results indicate that the areas with high entropy generation are spotted at the boundary layer around the blades, blade trailing edge, and volute region. Dou and Jiang ${ }^{18}$ applied the energy-gradient theory to analyze the flow instability in a centrifugal pump. Results showed that with the decrease of flow rate, the high energy loss was observed near the impeller exit and casing tongue. As for the experimental research, Wo and Bons ${ }^{19}$ investigated the off-design condition performance in a centrifugal pump experimentally.
Results indicated that the adverse flow near casing tongue and the destabilizing effect of diffusing pipe would induce the onset of surge. Krause et al. ${ }^{20}$ observed different stages of the rotating stall cells in a radial pump by using particle image velocimetry (PIV). He captured a spatially stable rotating stall cell under certain flow discharge, which started to rotate inside the impeller if the flow rate decreased further.

Although the experimental technologies have been successfully used in many studies, it is still inadequate to explore the mechanics of the flow instability by means of some traditional measurement technologies due to the complexity of fully developed turbulence. In the past century, some basic flow analysis such as transition from laminar to turbulence, shear flow, and boundary layer separation has been analyzed experimentally. ${ }^{21}$ Nevertheless, it is still tough to capture detailed information in some complex flow structures. With the development of CFD, numerical simulation of turbulent-separated flows has been approved to be a valuable tool in instability analysis. ${ }^{22}$ Although the low-cost Reynolds-averaged Navier-Stokes (RANS) models have been utilized successfully in many industrial configurations, it still shows defect in the accurate predictions in massively separated flows ${ }^{23}$ and could overestimate the viscous effects. ${ }^{24}$ Direct numerical simulations (DNS) resolve all the turbulence structures instead of modeling of them. Nevertheless, it is impractical to handle industry turbulence flow with $\mathrm{DNS}^{23}$ and the model is only applicable at low Reynolds numbers. ${ }^{25}$ Another kind of popular turbulence model is large-eddy simulation (LES), which resolves the large-scale structures and models the small-scale turbulence. Although more universal behavior is available, the formidably fine meshes and computational cost are too high for complex applications at high Reynolds numbers. ${ }^{25}$ Therefore, it turns to be a challenging task to choose an applicative turbulence closure method for a turbulence swirling flow. Several relevant researches on flow instability have been conducted with different turbulence closure models, including the realizable $k-\varepsilon$ model in analysis of flow in a double volute centrifugal pump, ${ }^{17} \mathrm{RNG} k-\varepsilon$ model utilized in swirling flow in a model combustor, ${ }^{26}$ SST $k-\omega$ model for swirling flows analysis, ${ }^{27}$ LES model applied in the cavitation analysis, ${ }^{28}$ and partially averaged NavierStokes (PANS) model used in simulations of flow in a draft tube of a Francis turbine. ${ }^{29}$

As mentioned above, PANS model is a relatively low-cost approach proposed recently by Girimaji, which is regarded as a bridge from RANS to DNS by two parameters: the unresolved-to-total ratios of kinetic energy $\left(f_{\mathrm{k}}\right)$ and dissipation $\left(f_{\varepsilon}\right){ }^{30}$ Girimaji performed a PANS model based on the $k-\varepsilon$ formulation for the simulation of flow past a circular cylinder with different value of $f_{\mathrm{k}}$. $^{31}$ The same simulation was also performed by Lakshmipathy and Girimaji ${ }^{32}$ with 
different value of $f_{\mathrm{k}}$ ranging from 0.5 to 1.0 in a lowReynolds number simulation. Results indicated that the predictions with small value of $f_{\mathrm{k}}$ coincide with the experimental data and LES results. Liu et al. ${ }^{33}$ performed a PANS model integrated by the RNG $k-\varepsilon$ model to investigate the flow influenced by the nonlinear shear stress. Results showed that the nonlinear PANS models were more reliable than linear PANS methods. Since Wilcox's $k-\omega$ model $^{34}$ behaves well in near-wall region, a PANS model based on the $k-\omega$ model was proposed by Lakshmipathy and Girimaji. ${ }^{35}$ Recently, Luo et al. ${ }^{23,36}$ developed a current PANS model based on the SST turbulence model ${ }^{37}$ with resolution control parameter $f_{\mathrm{k}}$ spatially varying and dynamically updated. Compared with the $k-\omega$ PANS model, the accuracy of SST PANS model better matches with the experimental data. In addition, the parameter $f_{\mathrm{k}}$ is always kept a constant value between zero and one in some conventional simulations, which represents that the PANS model would render to DNS and RANS, respectively. In order to ensure the predictions accuracy as high as possible according to the local grid distribution and turbulence information, a spatially varying approach on $f_{\mathrm{k}}$ was proposed by Girimaji and Abdol-Hamid, ${ }^{38}$ and it has been used extensively.

Inspired by those relevant researches, the objective in the current study is to validate the feasibility and accuracy of PANS model applying in the unsteady flow analysis for a centrifugal pump. In addition, the comparison has also been conducted to investigate the effect of inlet pipe with different geometry on hydraulic performances and the internal flow for centrifugal pumps, which has been seldom analyzed previously. Furthermore, based on the flow instability analysis in a straight inlet pipe, this paper intends to depict the relation between the internal flow and positive slope of pump performance. For better understanding, the energy loss analysis based on the energy equation is presented as well.

\section{Partially averaged Navier-Stokes model}

The incompressible Navier-Stokes equations for the instantaneous velocity $\left(V_{\mathrm{i}}^{*}\right)$ and pressure $(p)$ in a PANS model are given in equations (1) and (2)

$$
\begin{aligned}
& \frac{\partial V_{\mathrm{i}}}{\partial t}+V_{\mathrm{j}} \frac{\partial V_{\mathrm{i}}}{\partial x_{\mathrm{j}}}+\frac{\partial \tau\left(V_{\mathrm{i}}^{*}, V_{\mathrm{j}}^{*}\right)}{\partial x_{\mathrm{j}}}=-\frac{1}{\rho} \frac{\partial p}{\partial x_{\mathrm{i}}}+v \frac{\partial^{2} V_{\mathrm{i}}}{\partial x_{\mathrm{j}} \partial x_{\mathrm{j}}} \\
& -\frac{\partial^{2} p}{\partial x_{\mathrm{i}} \partial x_{\mathrm{i}}}=\frac{\partial V_{\mathrm{i}}}{\partial x_{\mathrm{j}}} \frac{\partial V_{\mathrm{j}}}{\partial x_{\mathrm{i}}}+\frac{\partial^{2} \tau\left(V_{\mathrm{i}}^{*}, V_{\mathrm{j}}^{*}\right)}{\partial x_{\mathrm{i}} \partial x_{\mathrm{j}}}
\end{aligned}
$$

where $V_{\mathrm{i}}$ is the partially averaged component and $v_{\mathrm{i}}$ is the total fluctuation component, shown in equation (3). The subfilter scale tensor $\tau\left(V_{\mathrm{i}}^{*}, V_{\mathrm{j}}^{*}\right)$ is a generalized central second moment

$$
V_{\mathrm{i}}^{*}=V_{\mathrm{i}}+v_{\mathrm{i}}
$$

Regarding the standard $k-\varepsilon$ model as a parent model of PANS, ${ }^{31}$ the turbulence closure equations are as follows

$$
\begin{aligned}
& \frac{\partial k_{\mathrm{u}}}{\partial t}+V_{\mathrm{j}} \frac{\partial k_{\mathrm{u}}}{\partial x_{\mathrm{j}}}=P_{\mathrm{u}}-\varepsilon_{\mathrm{u}}+\frac{\partial}{\partial x_{\mathrm{j}}}\left(\frac{v_{\mathrm{u}}}{\sigma_{\mathrm{ku}}} \frac{\partial k_{\mathrm{u}}}{\partial x_{\mathrm{j}}}\right) \\
& \frac{\partial \varepsilon_{\mathrm{u}}}{\partial t}+V_{\mathrm{j}} \frac{\partial \varepsilon_{\mathrm{u}}}{\partial x_{\mathrm{j}}}=C_{\mathrm{e} 1} \frac{P_{\mathrm{u}} \varepsilon_{\mathrm{u}}}{k_{\mathrm{u}}}-C_{\mathrm{e} 2}^{*} \frac{\varepsilon_{\mathrm{u}}^{2}}{k_{\mathrm{u}}}+\frac{\partial}{\partial x_{\mathrm{j}}}\left(\frac{v_{\mathrm{u}}}{\sigma_{\varepsilon \mathrm{u}}} \frac{\partial \varepsilon_{\mathrm{u}}}{\partial x_{\mathrm{j}}}\right)
\end{aligned}
$$

where $P_{\mathrm{u}}$ is unresolved production term of $k_{\mathrm{u}}$, similar with the corresponding RANS calculation. The values of the model constants are $C_{\varepsilon 1}=1.44, C_{\varepsilon 2}=1.92$, $\sigma_{\mathrm{k}}=1.0$, and $\sigma_{\varepsilon}=1.3 .{ }^{39}$ Some other parameters are

$$
\sigma_{\mathrm{ku}}=\sigma_{\mathrm{k}} \frac{f_{\mathrm{k}}^{2}}{f_{\varepsilon}} ; \quad \sigma_{\varepsilon \mathrm{u}}=\sigma_{\varepsilon} \frac{f_{\mathrm{k}}^{2}}{f_{\varepsilon}} ; \quad C_{\mathrm{e} 2}^{*}=C_{\mathrm{e} 1}+\frac{f_{\mathrm{k}}}{f_{\varepsilon}}\left(C_{\mathrm{e} 2}-C_{\mathrm{e} 1}\right)
$$

Two non-dimensional parameters $f_{\mathrm{k}}$ and $f_{\varepsilon}$ represent the unresolved-to-total ratios of kinetic energy and dissipation, respectively, which are defined as

$$
f_{\mathrm{k}}=\frac{k_{\mathrm{u}}}{k}, \quad f_{\varepsilon}=\frac{\varepsilon_{\mathrm{u}}}{\varepsilon}
$$

In high-Reynolds number simulations, the smallscale dissipation is unlikely to be resolved, ${ }^{29}$ and $f_{\varepsilon}$ is set to 1.0. However, it is difficult to determine the optimum value of the $f_{\mathrm{k}}$ in advance. Girimaji and Abdol-Hamid $^{38}$ proposed a spatially varying function in equation (8) to determine $f_{\mathrm{k}}$ upon the local grid size and turbulence length scale, which is similar to Kolmogorov scale ${ }^{38}$

$$
f_{\mathrm{k}}(x)=\frac{1}{\sqrt{C_{\mu}}}\left(\frac{\Delta}{\Lambda}\right)^{\frac{2}{3}}
$$

where $C_{\mu}$ is a model coefficient and $\Lambda$ is the Taylor turbulence length scale, which is defined by $\Lambda=k^{1.5} / \varepsilon$, and $\Delta$ stands for the local grid size defined as $\Delta=\left(\Delta x^{*} \Delta y^{*} \Delta z\right)^{1 / 3}$

To guarantee the value of $f_{\mathrm{k}}$ between 0 and $1,{ }^{29} \mathrm{a}$ modified formulation for $f_{\mathrm{k}}$ is shown in equation (9)

$$
f_{\mathrm{k}}(x)=\min \left(1, \frac{1}{\sqrt{C_{\mu}}}\left(\frac{\Delta}{\Lambda}\right)^{\frac{2}{3}}\right)
$$

\section{Calculation methods}

\section{Computation domain}

In a previous research, ${ }^{6}$ a tested pump with low specific speed was investigated previously using RANS model coupling with SST $k-\omega$ turbulence model to 
investigate the flow features in centrifugal pumps. Due to the non-straight inlet pipe of the tested pump shown in Figure 1, its internal flow is complicated, and the recirculation, flow separation, and rotating stall cells can be observed under part-load conditions. According to the numerical results, the flow upstream the impeller inlet of the tested pump is non-uniform and is suspected to affect the flow instability in the pump. In order to investigate the relationship between the positive slope phenomenon and rotating stall cells more clearly, a comparative model with a straight inlet pipe is treated in this study to achieve a uniform flow upstream impeller inlet. Note that the comparative model and the tested pump have the same geometry except for inlet pipe, which is non-straight inlet for the tested pump and straight inlet for the comparative model. For convenience, the tested pump having non-straight inlet pipe is named as Pump A and it is shown in Figure 1(a), and the comparative model having straight inlet pipe is named as Pump $\mathrm{A}^{\prime}$ and it is shown in Figure 1(b).
In the current paper, PANS method derived the standard $k-\varepsilon$ model is employed for simulating the flows in two centrifugal pumps with different inlet pipes to capture the effect of the swirl flow upstream impeller inlet. After mesh independence examination, each computational domain is discretized with about 3.2 million hexahedral cells. The mesh generated for all components is presented in Figure 2. Grid is refined in the areas such as near-wall and the expansion region.

\section{Boundary conditions}

For boundary conditions, mass flow rate is prescribed at domain inlet, and static pressure is set according to the experimental data at domain outlet. No-slip condition, $V_{i}^{*}=0$, is imposed on all solid walls. A time step of $3.33 \times 10^{-4} \mathrm{~s}$, corresponding to the runnerrotating angle of $2^{\circ}$ per time step, is applied for unsteady calculations. In addition, the max residuals for numerical convergence of the unsteady calculation

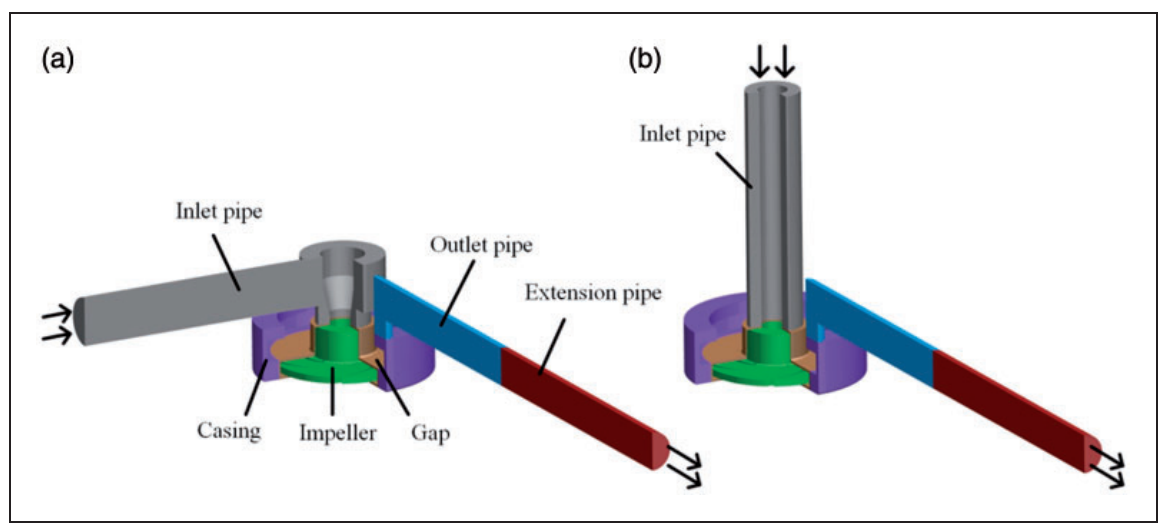

Figure I. Computational domains for two centrifugal pumps with different inlet pipes. (a) Pump A; (b) Pump A'.

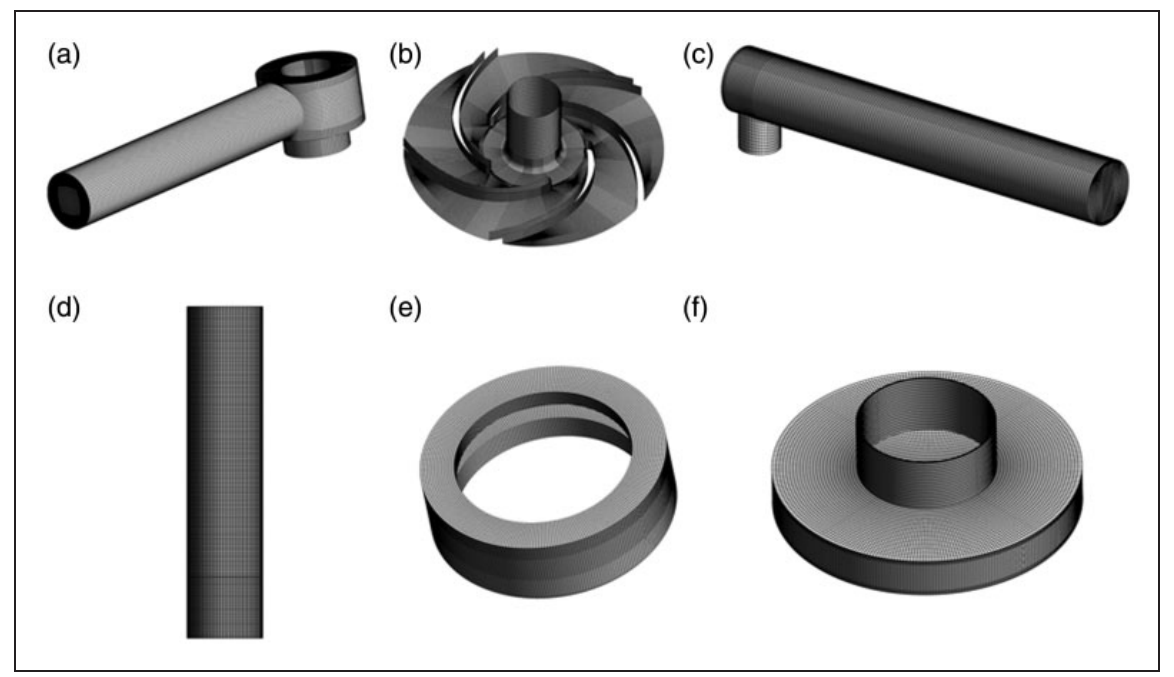

Figure 2. Mesh generation for all components: (a) inlet pipe (Pump A); impeller; (c) outlet pipe; (d) inlet pipe (Pump A'); (e) casing; (f) gap. 
at any flow rate are set below $10^{-6}$. Figure 3 gives the distribution of the mass pressure imbalance at the inlet domain under the condition of $0.78 \phi_{\text {bep }}\left(\phi_{\text {bep }}\right.$ represents the best efficient point condition) to explain the numerical convergence further. It can be noted that the relative error of the mass pressure imbalance is below $0.1 \%$, which is regarded small enough to reach a convergence.

Numerical simulations are completed using the commercial CFD code ANSYS-CFX.

\section{Results and discussion}

In this section, the performance comparisons are first conducted to validate the capacities of the current PANS model for predicting the flow instability in the pump. Then the detailed flows in the impeller inlet, impeller, and outlet pipe are discussed as follows.

\section{Performance comparisons}

Pump A was installed and tested at the Key Laboratory of Fluid Transmission Technology of Zhejiang province, China. Main geometrical parameters for the pump are shown in Table 1. More

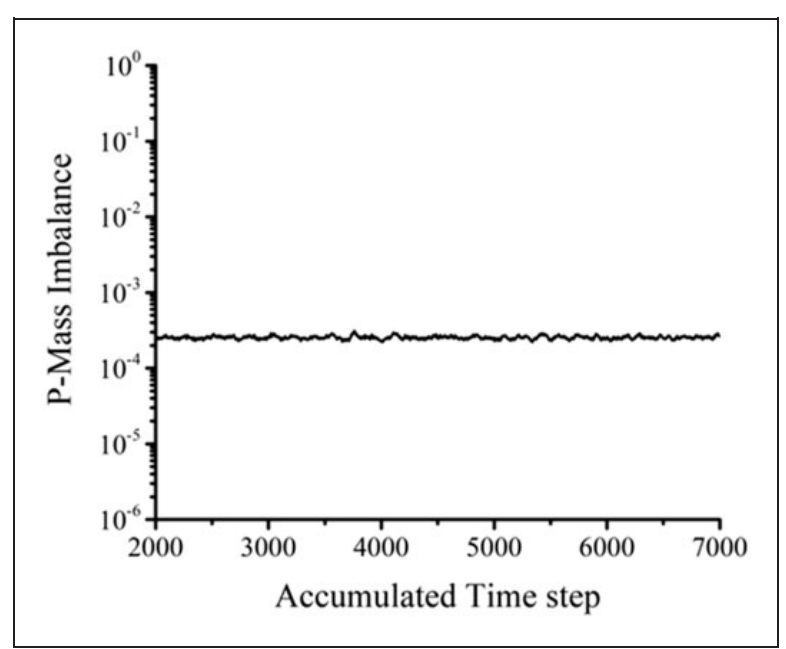

Figure 3. Mass pressure imbalance distribution at the inlet domain of the Pump $\mathrm{A}^{\prime}$.

Table I. Main parameters of the Pump A.

\begin{tabular}{ll}
\hline Parameter & Value \\
\hline Impeller inlet diameter $D_{1}$ & $56 \mathrm{~mm}$ \\
Impeller exit diameter $D_{2}$ & $142 \mathrm{~mm}$ \\
Blade number & 5 \\
Shaft rotational speed $n$ & $1000 \mathrm{r} / \mathrm{min}$ \\
Flow rate at design point $Q_{d}$ & $1.8 \mathrm{~m}^{3} / \mathrm{h}$ \\
Pump head at design point $H_{\mathrm{d}}$ & $2.67 \mathrm{~m}$ \\
\hline
\end{tabular}

information can be found in Ye et al. ${ }^{6}$ The experimental data of the Pump A are available for comparison.

Several dimensionless parameters, head coefficient $\psi$, flow coefficient $\phi$, pump efficiency $\eta$, and power coefficient $\tau_{\text {in }}$, have been defined as shown in equations (10) to (13)

$$
\begin{aligned}
& \phi=\frac{Q}{2 \pi R_{2} b_{2} u_{2}} \\
& \psi=\frac{H}{u_{2}^{2} /(2 g)} \\
& \eta=\frac{\rho g H Q}{P_{\text {in }}} \\
& \tau_{\text {in }}=\frac{P_{\text {in }}}{\left(\rho \pi R_{2} b_{2} u_{2}^{3}\right) / 2}
\end{aligned}
$$

where $Q$ represents flow rate, $H$ is pump head, and $P_{\text {in }}$ is power input to pump shaft. $R_{2}, b_{2}$, and $u_{2}$ are the radius, blade width, and peripheral velocity at the impeller exit, respectively.

The characteristic curves of two pumps are displayed in Figure 4. Note that the scattered black marks are experimental data for Pump A, the red lines are numerical results for Pump A, and the blue lines stand for numerical results for Pump $\mathrm{A}^{\prime}$. Several typical operation conditions are marked as $\mathrm{OP}_{\mathrm{A}} \sim \mathrm{OP}_{\mathrm{E}}$ in Figure 4(a), where $\mathrm{OP}_{\mathrm{A}}$ represents the operation at $\phi=0.0113=0.5 \phi_{\text {bep }}, \mathrm{OP}_{\mathrm{B}}$ is $\phi=0.015=$ $0.67 \phi_{\text {bep }}, \mathrm{OP}_{\mathrm{C}}$ is $\phi=0.0163=0.72 \phi_{\text {bep }}, \mathrm{OP}_{\mathrm{D}}$ represents $\phi=0.0175=0.78 \phi_{\text {bep }}$, and $\mathrm{OP}_{\mathrm{E}}$ represents $\phi=$ 0.0225 . Note that the best efficiency point locates at $\phi_{\text {bep }}=0.0225$ for the Pump A based on the experimental data.

As shown in Figure 4, there is a reasonable agreement between the numerical and experimental data for Pump A. The positive slopes are perfectly captured at part-load conditions, e.g. $\mathrm{OP}_{\mathrm{A}}, \mathrm{OP}_{\mathrm{B}}$, and $\mathrm{OP}_{\mathrm{D}}$, the same operation conditions measured by experimental data. ${ }^{6}$ The maximum relative error for head coefficient is $4.27 \%$ under the operation condition of $\phi=0.0100$, and the minimum relative error is $0.17 \%$ under condition of $\phi=0.0175$. As for the efficiency prediction, the maximum deviation is about $6.79 \%$ under condition of $\phi=0.0138$, and the minimum deviation is about $2.97 \%$ at the best efficiency point condition. It should be noted that the mechanical loss such as friction loss is neglected in the simulation. Thus, it is reasonable that the predicted head or efficiency is relatively higher than the experimental data. Therefore, the numerical results are not perfect but still acceptable for the current research.

The results displayed in Figure 4 indicate that head coefficient and efficiency of the Pump A' are slightly larger than that of the Pump A under the operation of $\mathrm{OP}_{\mathrm{E}}$. This is because the energy loss in the nonstraight inlet pipe of the Pump A is higher than that 


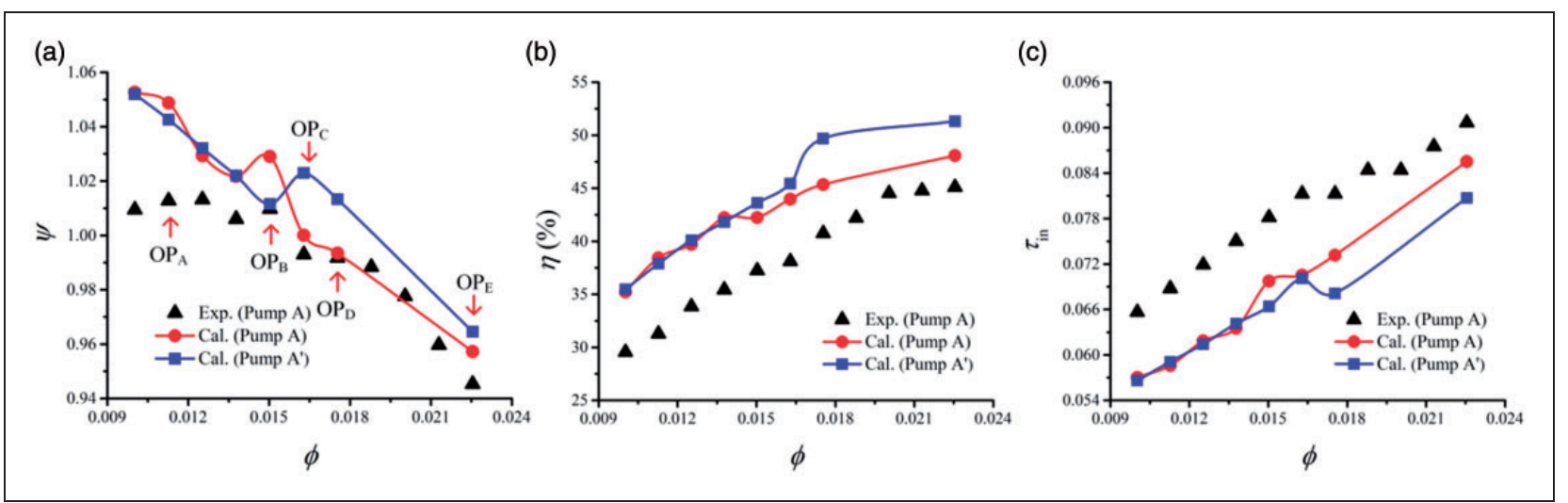

Figure 4. Characteristic curves of two pumps: (a) $\psi-\phi$; (b) $\eta-\phi$; (c) $\tau_{\text {in }}-\phi$.

in Pump $\mathrm{A}^{\prime}$. The same phenomenon can be also captured from $\mathrm{OP}_{\mathrm{C}}$ to $\mathrm{OP}_{\mathrm{E}}$, where the input power of Pump A is larger, and efficiency is lower than that of Pump $\mathrm{A}^{\prime}$. At the operation of $\mathrm{OP}_{\mathrm{B}}$, which locates at the positive slope for the Pump $A^{\prime}$, pump head becomes different for two pumps. Around the operation of $\mathrm{OP}_{\mathrm{A}}$, the hydraulic performances are almost the same in both pumps. These results indicate that increasing the flow rate may raise radial pressure gradient at the section of non-straight pipe inlet (Pump $\mathrm{A}^{\prime}$ ), which can cause secondary flow. In addition, this condition produces undesired flow at the pump impeller inlet and, consequently, has negative effects on pump performance.

Figure 5 shows the comparisons of the internal flow in the pump impellers under three part-load conditions, i.e. $\phi=0.5 \phi_{\text {bep }}, \phi=0.6 \phi_{\text {bep }}$, and $\phi=0.78 \phi_{\text {bep }}$. Note that the images at left column are PIV results, and the middle ones are numerical results for Pump A. The right pictures are numerical results for Pump $\mathrm{A}^{\prime}$ in order to compare the different flow features resulted from the different geometries of inlet pipe.

As for the results of Pump A, although the scale of separation flow is a little over-estimated by the numerical simulation, it is believed that the averaged flow such as velocity contour and streamlines on the midspan section of the impeller blade is in good accordance with the measured data. These results confirm that current PANS model is suitable to predict the internal flow in a centrifugal pump. Flow separation occurs at each blade suction side owing to large incidence angles. ${ }^{6}$ Though the streamlines seem somehow smoother with the increase of flow coefficient, e.g. the flow in blade-to-blade passage A, flow separation still remains strong in the impeller under three part-load conditions.

Due to the different geometries of inlet pipe, the velocity distributions and streamlines of the Pump $\mathrm{A}^{\prime}$ are different from that of Pump A. Under the condition of $\phi=0.5 \phi_{\text {bep }}$, all blade-to-blade passages for the Pump A are blocked off by the flow separation, while the flow separation at blade-to-blade passage A and B is very slight for Pump $\mathrm{A}^{\prime}$. Under the condition of $\phi=0.78 \phi_{\text {bep }}$, the flow separation at blade-to-blade passage $A$ for the Pump $A^{\prime}$ is much slighter than that for Pump A. However, the large-scale flow separation still occurs in other blade-to-blade passages, and the flow instability is basically the same for both pumps. Detailed comparisons will be carried out in the following texts.

Further comparison is carried out for the absolute circumferential velocity profile under the operation condition of $\mathrm{OP}_{\mathrm{D}}$ along the reference line $\mathrm{S} 1$, which is very close to the blade pressure surface in bladeto-blade passage A as shown in Figure 5. Note that $r / R=1$ means the location at impeller exit. Since the condition of $\mathrm{OP}_{\mathrm{D}}$ is the operation having positive slope, the flow in the pump impeller is unstable, and there is almost the largest discrepancy between the simulation and the experiment for the pump performance (shown in Figure 4(a)). However, the predicted tendency of the velocity profiles shows good accordance with the experimental data. As shown in Figure $6, v_{\mathrm{u}}$ increases along the radial direction and drops near the impeller exit due to the presence of flow separation. Compared with the steep drop of $v_{\mathrm{u}}$ for Pump A, the drop of $v_{\mathrm{u}}$ is alleviated at impeller exit for the Pump $\mathrm{A}^{\prime}$.

Basing on these comparisons, the present simulation accuracy is acceptable. Further improvement will be carried out in the further study.

\section{Impeller inlet conditions}

Having validated the capacities of the current PANS model, detail analyses on the impeller inlet are carried out in this subsection.

Since the Batchelor vortex could satisfactorily represent the velocity field of trailing vortices superimposed axial flow, ${ }^{10}$ the swirl flow in the draft tube of a Francis turbine was successfully investigated. ${ }^{40}$ Similar to a draft tube, impeller inlet is also the stationary component for turbomachines. Despite researches on the swirl flow in the inlet pipe are seldom reported, the averaged velocity profiles, except for near-wall shear layer, can be approximated by Batchelor vortex family. 


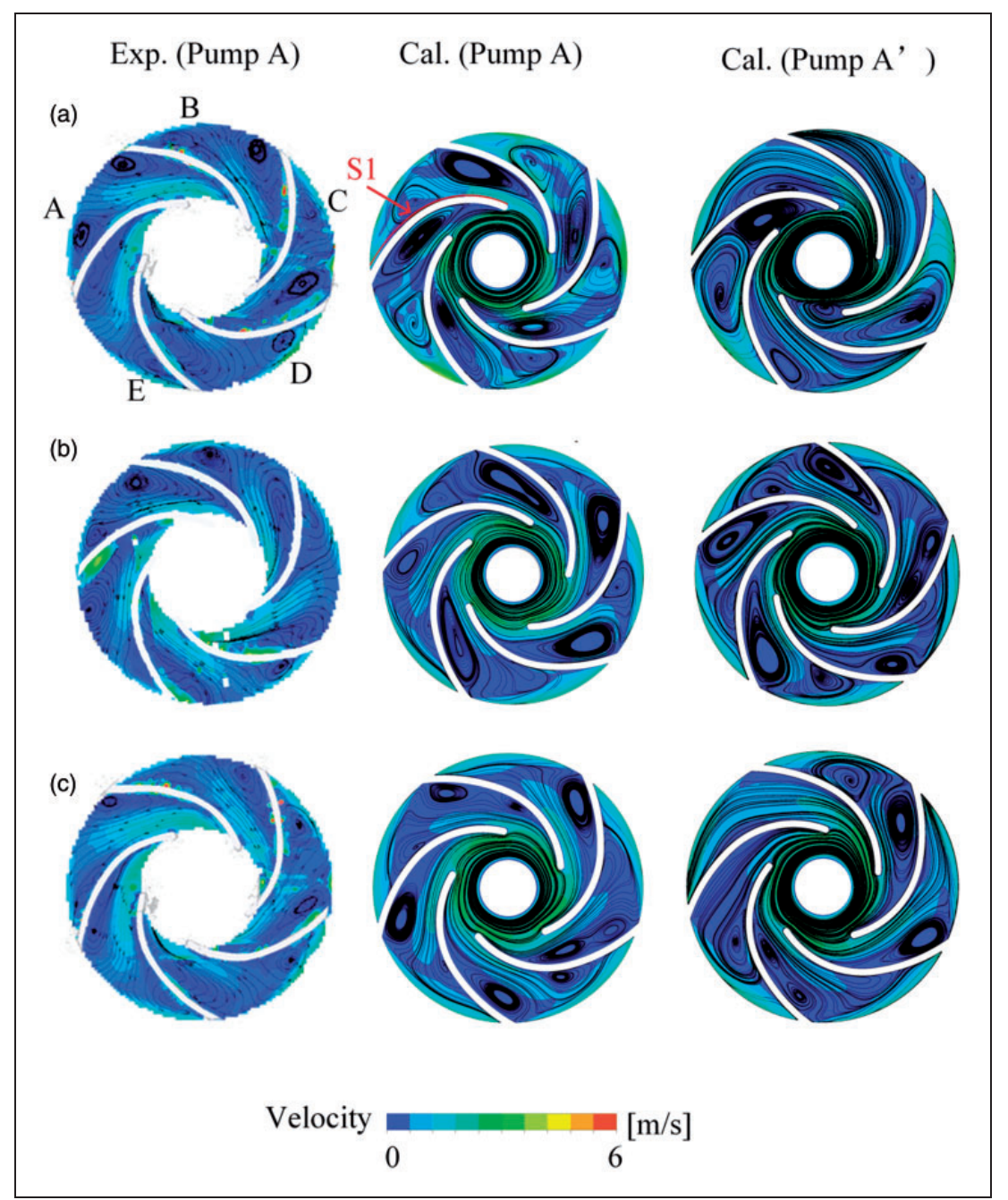

Figure 5. Velocity contour and streamlines on mid-span section under three part-load conditions: (a) $\phi=0.5 \phi_{\text {bep }}$; (b) $\phi=0.6 \phi_{\text {bep }}$; (c) $\phi=0.78 \phi_{\text {bep }}$

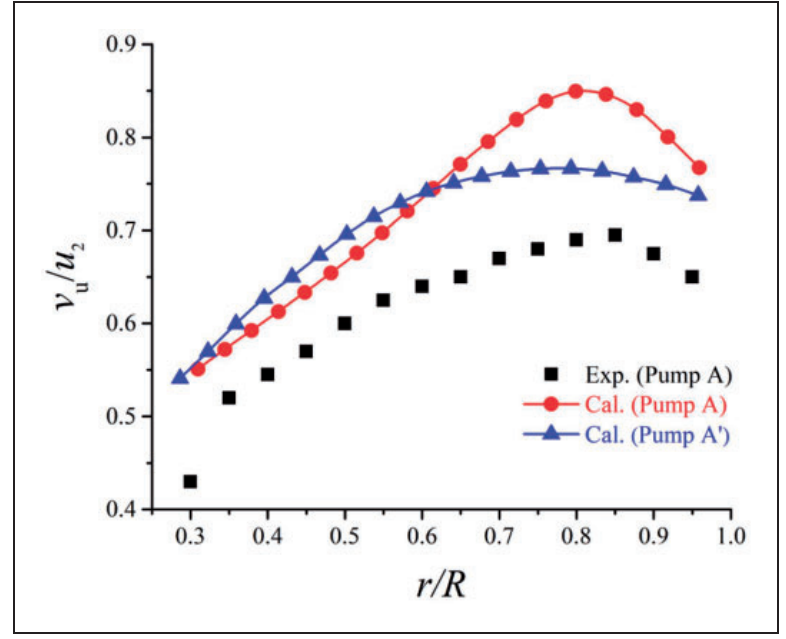

Figure 6. Distributions of absolute circumferential velocity.

In a cylindrical coordinate $\left(r^{*}, \theta^{*}, z^{*}\right)$ (for coordinate $\mathrm{z}^{*}$, downstream is defined as the positive direction), the similarity solution of velocity profiles is given by equations (14) to (16), representing the radial, azimuthal, and axial velocity components, respectively

$$
\begin{aligned}
& U^{*}=U^{*}\left(r^{*}\right)=0 \\
& V^{*}=V^{*}\left(r^{*}\right)=\frac{\Omega_{\mathrm{c}}^{*} R^{*}}{r^{*} / R^{*}}\left[1-e^{-\left(r^{*} / R^{*}\right)^{2}}\right] \\
& W^{*}=W^{*}\left(r^{*}\right)=W_{\infty}^{*}+\left(W_{\mathrm{c}}^{*}-W_{\infty}^{*}\right) e^{-\left(r^{*} / R^{*}\right)^{2}}
\end{aligned}
$$

where $W_{\infty}^{*}$ is the axial velocity at the inlet section of inlet pipe, $W_{\mathrm{c}}^{*}$ is axial velocity along the centerline, $\Omega_{\mathrm{c}}^{*}$ is the rotation rate along the axis, and $R^{*}$ is radius of vortex core.

As mentioned by Lessen et al., ${ }^{11}$ the non-dimensional mean velocity profiles can be transferred to equations (17) to (19)

$$
\begin{aligned}
& U=U(r)=0 \\
& V=V(r)=\frac{q}{r}\left[1-e^{-r^{2}}\right] \\
& W=W(r)=a+e^{-r^{2}}
\end{aligned}
$$


where $r=r^{*} / R^{*}, \quad q=\left(\Omega_{\mathrm{c}}^{*} R^{*}\right) / \Delta W^{*}, \quad a=W_{\infty}^{*} / \Delta W^{*}$, and $\Delta W^{*}=W_{\mathrm{c}}^{*}-W_{\infty}^{*}$. Specially, $q$ and $a$ are the swirl ratio and external axial velocity.

According to Zhang et al.'s work, ${ }^{3}$ the flow is a coflowing jet if $a>0$, a coflowing wake if $a<-1$, and a counterflowing jet or wake if $-1<a<0$. The flow is under absolute instability (i.e. AI) region if the counterflowing jet or wake occurs, which indicates that the perturbation will propagate upstream or downstream, resulting in absolute instability. For cases of $a>0$ and $a<-1$, the flow is under convective instability $(\mathrm{CI})$ region.

Figure 7 defines a reference section perpendicular to pump axis (marked by "RP") and a reference line parallel to pump axis (marked by "RL"). These will be used for analysis in the following text.

Figure 8 shows the distribution of parameter $a$ and axial vorticity coefficient $\omega_{\mathrm{z}}$ profiles along RL under two conditions in both pumps. The non-dimensional parameter $\omega_{\mathrm{z}}$ is defined as

$$
\omega_{z}=\frac{2 \Omega_{c}^{*} D^{*}}{V_{0}^{*}} e^{-r^{2}}
$$

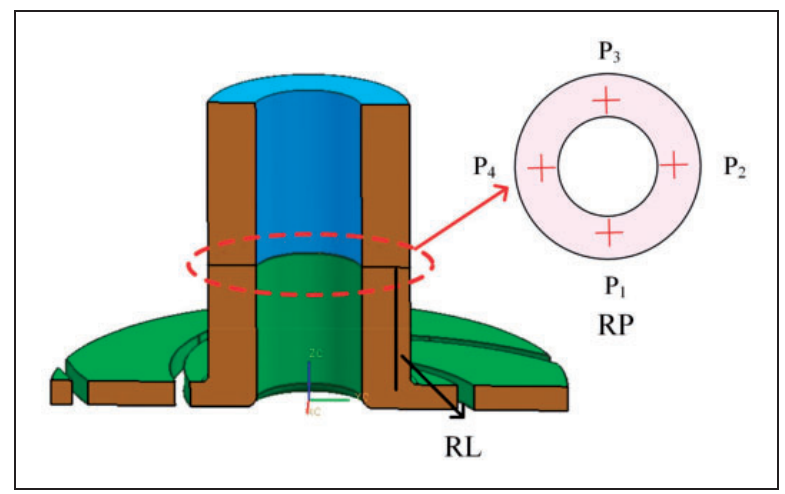

Figure 7. Location of the reference section and reference line. where $D^{*}$ is diameter of inlet tube, velocity scale $V_{0}^{*}$ is the averaged velocity at the inlet tube, i.e. $V_{0}^{*}=4 Q^{*} /$ $\pi D^{* 2}$, and $Q^{*}$ is the volume flow rate.

The results in Figure 8 depict that the Batchelor vortex in the inlet pipe is always in the CI region because the values of the parameter $a$ always remain positive in both two part-load conditions, $\mathrm{OP}_{\mathrm{A}}$ and $\mathrm{OP}_{\mathrm{D}}$. This indicates that the instability in the inlet pipe would not propagate both upstream and downstream. In both pumps, the axial vorticity $\omega_{\mathrm{z}}$ is induced by the "pre-swirling" effect by the rotation of the impeller. Further, the axial vorticity $\omega_{z}$ increases as the fluid flows downstream under the condition of $\mathrm{OP}_{\mathrm{D}}$. The tendency of the parameter $a$ and axial vorticity coefficient $\omega_{z}$ are almost the same under the condition of $\mathrm{OP}_{\mathrm{D}}$. That means the flow in the inlet pipe is more affected by the unstable flow in pump impeller during this operation condition. However, under the condition of $\mathrm{OP}_{\mathrm{A}}$, the averaged value of axial vorticity coefficient in the Pump $\mathrm{A}$ is larger than that in the Pump $\mathrm{A}^{\prime}$. This is due to different geometries of the inlet pipe.

Further research on the pressure oscillations under the condition of $\mathrm{OP}_{\mathrm{D}}$ in Pump $\mathrm{A}^{\prime}$ is carried out in Figure 9. The corresponding monitoring points are defined in RP section in Figure 7. In addition, the non-dimensional parameter, pressure coefficient, is defined in equation (21)

$$
C_{\mathrm{p}}=\frac{p_{\mathrm{i}}-\bar{p}}{0.5 \rho u_{2}^{2}}
$$

where $p_{\mathrm{i}}$ presents the instantaneous value of static pressure at a monitoring point and $\bar{p}$ is the averaged pressure.

As shown in Figure 9, the results indicate that the dominate frequency in Pump $\mathrm{A}^{\prime}$ is the same as the frequency of impeller rotation, $f_{\mathrm{n}}$. Thus, the low-
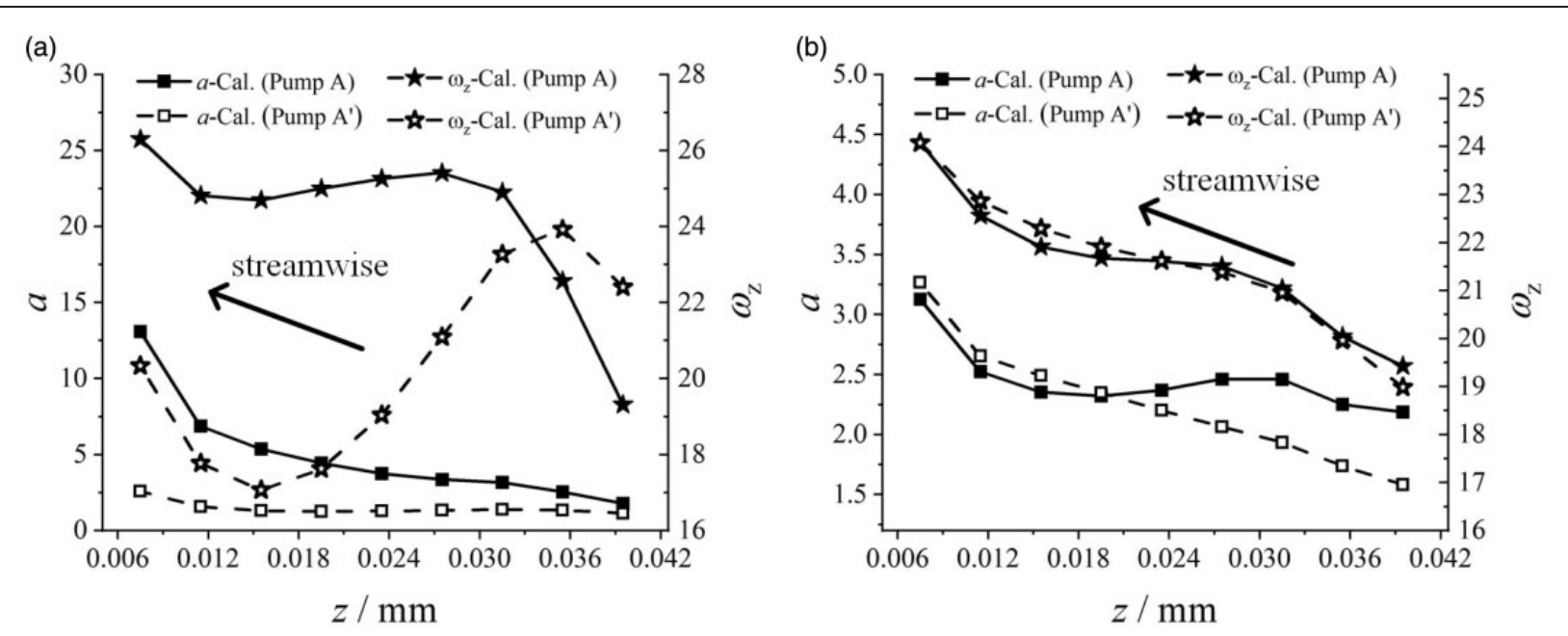

Figure 8. Distributions of external axial velocity and axial vorticity coefficient of Batchelor vortex under two part-load conditions: (a) $\mathrm{OP}_{\mathrm{A}}$; (b) $\mathrm{OP}_{\mathrm{D}}$. 
frequency pressure oscillation induced by rotating stall $^{6}$ in the impeller does not occur in the inlet pipe, and the internal flow in the straight inlet pipe is under stable state. However, the results of the pressure oscillations for the Pump A are much more complicated, with lower frequency components (not shown here).

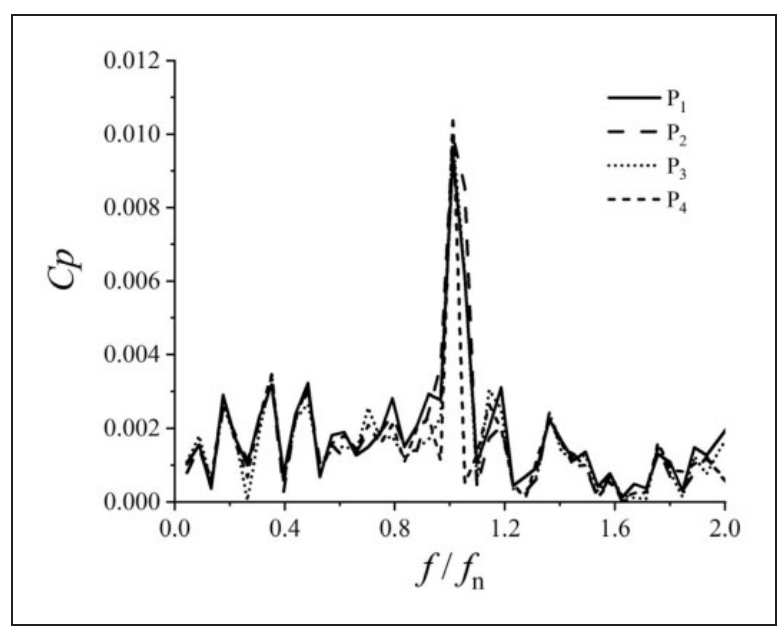

Figure 9. Pressure fluctuations at monitoring points under condition of $\mathrm{OP}_{\mathrm{D}}$.
Hence, straight inlet pipe may eliminate the unstable flow upstream the impeller. This fact is helpful to reveal the mechanism of flow instability in the impeller more clearly.

\section{Impeller}

Since the flow instability analysis in the impeller of the Pump A has been investigated in our previous research, ${ }^{6}$ the instability characteristics in the Pump $\mathrm{A}^{\prime}$ is mainly discussed in this section to conclude the interaction of positive slope phenomenon and internal flow information with different geometries of the inlet pipe.

Propagation of rotating stall cells. Under part-load conditions, the propagation of rotating stall cells is quite common in guide vanes and blade-to-blade passages for centrifugal pumps. To illustrate the evolution of rotating stall cells in Pump $\mathrm{A}^{\prime}$, the unsteady results of the flow coefficient $\phi$ during impeller rotation under four part-load conditions are displayed in Figure 10. Some instantaneous vorticity-z contours at the midspan section are also shown in Figure 11. Note that the $T_{\mathrm{n}}$ in the figures means one period of impeller rotation, and the locations of blade-to-blade passage

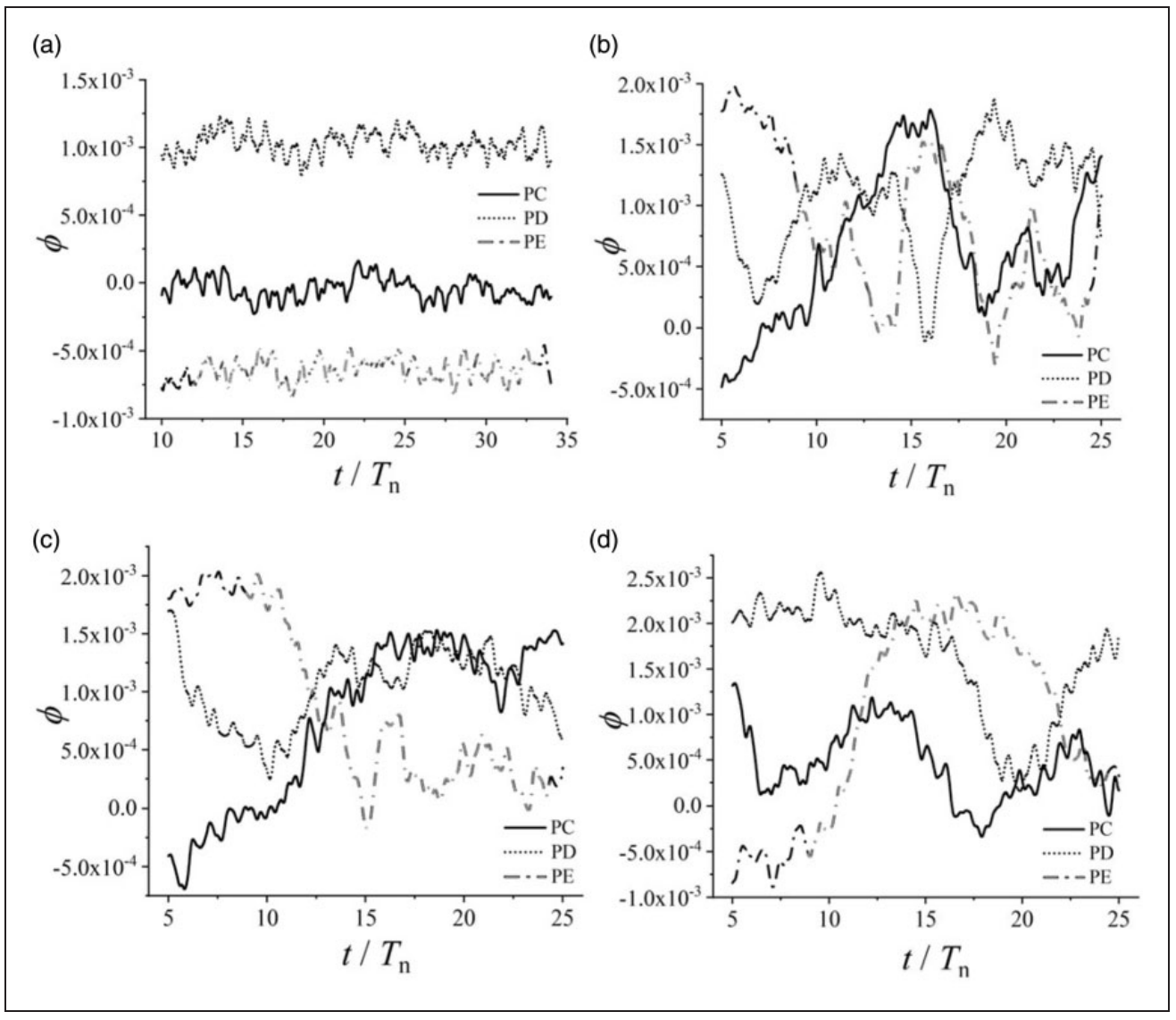

Figure 10. Temporal evolution of flow coefficient in Pump $A^{\prime}$ under four typical part-load conditions: (a) $O P_{A}$; (b) $O P_{B}$; (c) $O P_{C}$; (d) $O P_{D}$. 


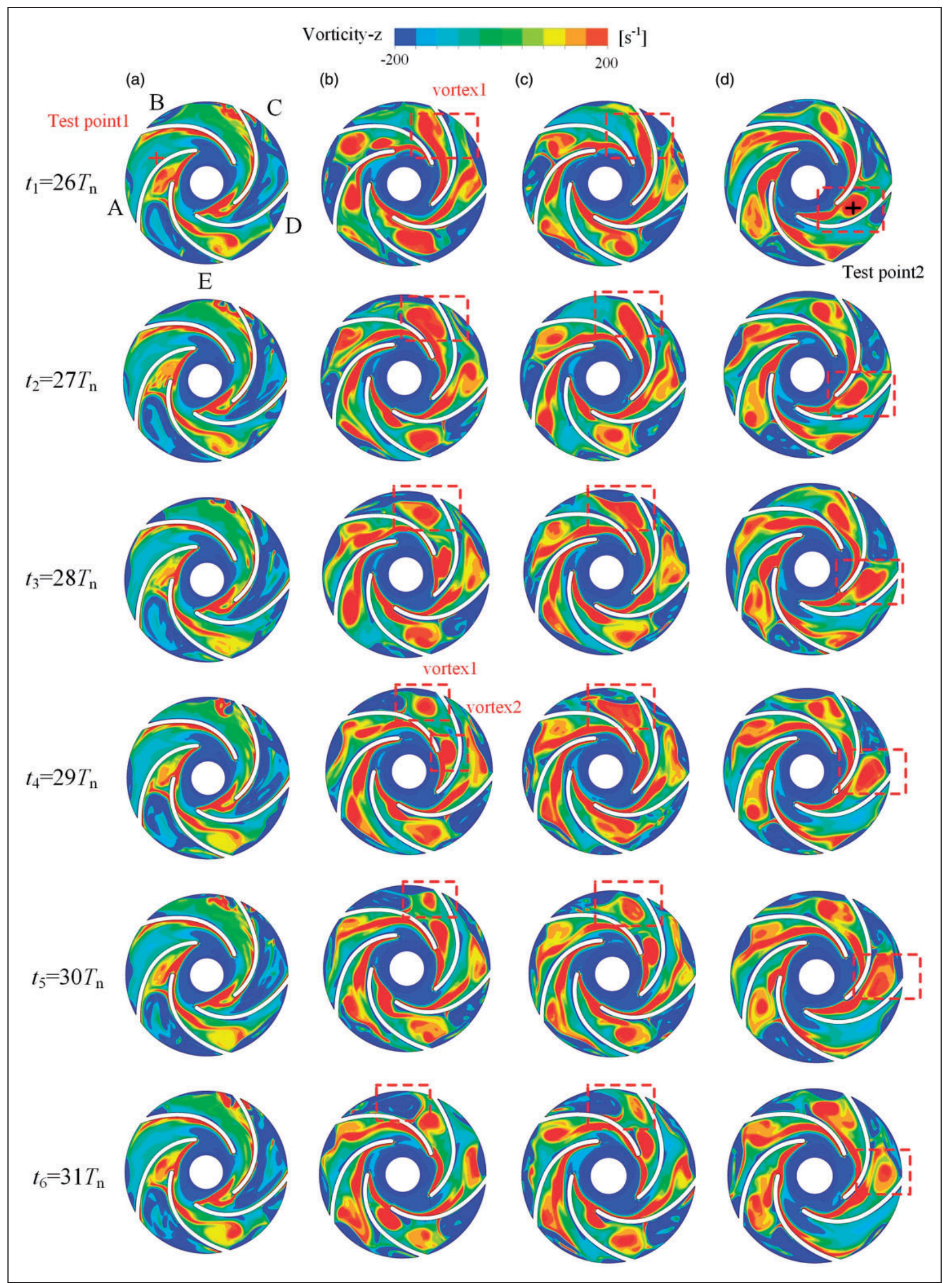

Figure II. Instantaneous vorticity-z at mid-span section in Pump $A^{\prime}$ under four typical conditions: (a) $O P_{A}$; (b) $\mathrm{OP}_{B}$; (c) $\mathrm{OP}_{C}$; (d) $O P_{D}$. 
A-E are clarified in Figure 5. In the following text, blade-to-blade passages $\mathrm{C}, \mathrm{D}$, and $\mathrm{E}$ are simplified as $\mathrm{PC}, \mathrm{PD}$, and $\mathrm{PE}$, respectively.

The following can be observed as shown in Figure 10:

1. Under the condition of $\mathrm{OP}_{\mathrm{A}}$, the flow rate coefficient $\phi$ in the blade-to-blade passage PC is near zero due to the great blockage by the flow separations as shown in Figure 5(a). The flow rate in the blade-to-blade passage PD is always positive, while that in the blade-to-blade passage, $\mathrm{PE}$ is reverse flow. Thus, the flow at this operation is relatively stable.

2. Under three other conditions, the flow in all bladeto-blade passages oscillates greatly, and the flow oscillation is the largest under the operation $\mathrm{OP}_{\mathrm{D}}$, which means the internal flows are unstable under those operations. The reverse flow shifts in different blade-to-blade passages at different time instants. For $\mathrm{OP}_{\mathrm{B}}$, the reverse flow first occurs in the blade-to-blade passage PC. After several periods of impeller revolution, the reverse flow with small-scale occurs in the blade-to-blade passage $\mathrm{PE}$ and $\mathrm{PD}$ in sequence.

3. It is clear that the reverse flow with large-scale does not occur in the same blade-to-blade passage. Under the condition of $\mathrm{OP}_{\mathrm{A}}$, there is a reverse flow in blade-to-blade passage PE. Under the operation conditions of $\mathrm{OP}_{\mathrm{B}}$ and $\mathrm{OP}_{\mathrm{C}}$, the reverse flow occurs in the blade-to-blade passage PC. Under the operation $\mathrm{OP}_{\mathrm{D}}$, the reverse flow occurs in the blade-to-blade passage PE.

Figure 11 shows the impeller flow visualized by instantaneous axial vorticity distribution on the midspan section of impeller under four typical conditions, from $\mathrm{OP}_{\mathrm{A}}$ to $\mathrm{OP}_{\mathrm{D}}$. Five impeller revolution circles ranging from $t=26 T_{\mathrm{n}}(1.56 \mathrm{~s})$ to $t=31 T_{\mathrm{n}}(1.86 \mathrm{~s})$ are selected for analysis.

It is noted that under the condition of $\mathrm{OP}_{\mathrm{A}}$, the blade-to-blade passage PE is totally blocked off near blade leading edge by vortices, and the blockage remains almost unchanged during this period as shown in Figure 10(a). Meanwhile, the blockage by vortices is also severe in the blade-to-blade passage PC under this operation. Results clearly observe the revolution of vortical flow in the blade-to-blade passage under three conditions: $\mathrm{OP}_{\mathrm{B}}, \mathrm{OP}_{\mathrm{C}}$, and $\mathrm{OP}_{\mathrm{D}}$. Under these conditions, the size and position of the vortices are marked in a rectangle box. Under the operation $\mathrm{OP}_{\mathrm{D}}$, the periodic state of rotating stall cells in the blade-to-blade passage PC captured by the unsteady numerical calculation can be divided into several typical stages, i.e. vortex inception $\left(t_{1}=26 T_{\mathrm{n}}\right)$, development $\left(t=27 T_{\mathrm{n}}, t=28 T_{\mathrm{n}}\right)$, split $\left(t=29 T_{\mathrm{n}}\right)$, decay $\left(t=30 T_{\mathrm{n}}\right)$, and shedding $\left(t=31 T_{\mathrm{n}}\right)$. The rotating stall behavior in Pump $\mathrm{A}^{\prime}$ shows the similar unstable flow feature with the results of Pump A. ${ }^{6}$ Thus, under three conditions having positive slope, the vortical flow in the impeller develops from $26 T_{\mathrm{n}}$ to $31 T_{\mathrm{n}}$.

It is well acknowledged that the propagation of rotating stall cells induces the pressure fluctuations with low frequency under unstable operation conditions.

The standard deviation of pressure fluctuations, which is defined in equation (22), is utilized for further investigating the pressure fluctuations intensity in the impeller. Results between two pumps are plotted in Figure 12

$$
C p_{\mathrm{sd}}=\frac{\sqrt{\frac{1}{N} \sum_{i=1}^{N}\left[p\left(x, y, z, t_{i}\right)-\overline{p(x, y, z, t)}\right]^{2}}}{\frac{1}{2} \rho u_{2}^{2} \pi D_{2} b_{2}}
$$

where $N$ is the number of time steps and $p\left(x, y, z, t_{\mathrm{i}}\right)$ is instantaneous pressure.

In our previous study, ${ }^{6}$ the conditions of $\mathrm{OP}_{\mathrm{A}}$ and $\mathrm{OP}_{\mathrm{D}}$ in Pump $\mathrm{A}$ are under the positive slope regions, whereas the condition of $\mathrm{OP}_{\mathrm{A}}$ in the Pump $\mathrm{A}^{\prime}$ is under the normal condition. As shown in Figure 12, the distributions of $C P_{\mathrm{sd}}$ in the Pump A are similar under the conditions of $\mathrm{OP}_{\mathrm{A}}$ and $\mathrm{OP}_{\mathrm{D}}$, indicating that strong pressure fluctuations are captured on the mid-span section of the impeller. However, the results in Pump $\mathrm{A}^{\prime}$ are much different. The intensity of $C P_{\text {sd }}$ under the condition having positive slope $\left(\mathrm{OP}_{\mathrm{D}}\right)$ is higher than that under normal condition $\left(\mathrm{OP}_{\mathrm{A}}\right)$. Thus, strong pressure fluctuation is a typical characteristic as the positive slope phenomenon occurs, and the pressure fluctuation is smaller under the relative stable condition, $\mathrm{OP}_{\mathrm{A}}$.

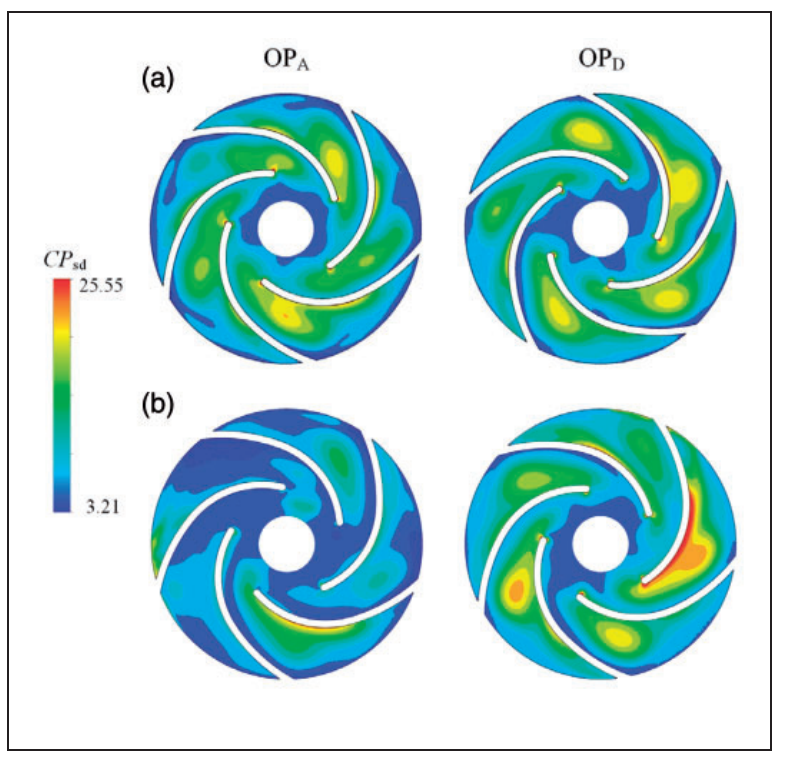

Figure 12. Standard deviation of pressure fluctuations on mid-span section under two part-load conditions: (a) Pump A; (b) Pump A'. 
Instability analysis based on Rayleigh-Taylor criterion. Rayleigh-Taylor (R-T) criterion $^{8}$ is one useful method to analyze flow instability, and it is suitable for the cases when the fluid rotates or encounters a curved surface. This kind of instability is named as "centrifugal instability." In this subsection, R-T criterion is utilized to investigate the flow instability under two typical conditions $\left(\mathrm{OP}_{\mathrm{A}}\right.$ and $\left.\mathrm{OP}_{\mathrm{D}}\right)$ in Pump A'.

For $\mathrm{R}-\mathrm{T}$ criterion, vortices in blade-to-blade passages are selected for analysis. The location of vortex core is named as "C." The radius of one initial fluid particle named as " $\mathrm{A}$ " in a vortex is $r_{1}$, and the angular speed is $\Omega_{1}$. At the other instant, the fluid particle propagates to the location " $\mathrm{B}$," and the radius and angular speed are $r_{2}$ and $\Omega_{2}$, respectively. A hypothesis for the inviscid flow is applied; if $r_{1}<r_{2},\left(\Omega_{1}\right.$ $\left.r_{1}^{2}\right)<\left(\Omega_{2} r_{2}^{2}\right)$, the vortex is in a stable state. Otherwise, the vortex is in an unstable state. If flow particles are under "centrifugal instability" state, they would propagate downstream due to the centrifugal force.

Figure 13 shows the vortices in the blade-to-blade passages under two typical conditions. In Figure 13(a), A1 is the fluid particle at one instant, B1 is the fluid particle at another instant, and $\mathrm{C} 1$ is the location of vortex core. As shown in Table 2, at the instant of $t=26 T_{\mathrm{n}}$ under condition of $\mathrm{OP}_{\mathrm{A}}$, it is noted that $r_{1}>r_{2}$, and $\left(\Omega_{1}\right.$ $\left.r_{1}^{2}\right)>\left(\Omega_{2} r_{2}^{2}\right)$. According to the criterion, this vortex is in a stable state; the fact has been confirmed by the above discussion. As for the vortex under the condition of $\mathrm{OP}_{\mathrm{D}}$, it is found that when $r_{1}<r_{2},\left(\Omega_{1} r_{1}^{2}\right)>\left(\Omega_{2} r_{2}^{2}\right)$. The results indicate that the vortex in the blade-toblade passage is the unstable state under the condition of $\mathrm{OP}_{\mathrm{D}}$. Thus, the flow instability in the present study is "centrifugal instability."

Unsteady radial forces. The radial forces have been calculated at each instant. The non-dimensional radial force coefficient $C_{f \mathrm{r}}$ is defined in equation (23)

$$
C_{f \mathrm{ri}}=\frac{\left[F_{\mathrm{x}}\left(t_{\mathrm{i}}\right)^{2}+F_{\mathrm{y}}\left(t_{\mathrm{i}}\right)^{2}\right]^{\frac{1}{2}}}{\frac{1}{2} \rho u_{2}^{2} \pi D_{2} b_{2}}
$$

where subscript $i$ means the $i$ th time step. $F_{\mathrm{x}}\left(t_{\mathrm{i}}\right)$ and $F_{\mathrm{y}}\left(t_{\mathrm{i}}\right)$ are instantaneous $x$ and $y$ components of radial force, respectively.

Figure 14 shows unsteady radial forces under two part-load conditions in the Pump $\mathrm{A}^{\prime}$ during nearly 26 impeller rotation cycles. At operation $\mathrm{OP}_{\mathrm{A}}$, the radial force oscillates periodically with the same cycle of impeller rotation. Under the condition of $\mathrm{OP}_{\mathrm{D}}$, the radial force oscillates with two different cycles: one is the same cycle of impeller rotation and another is much longer cycle due to the propagation of rotating stall cells. It is clear that the flow instability due to

Table 2. Instability analysis with R-T criterion under different conditions.

\begin{tabular}{lllll}
\hline Condition & $r_{1}$ & $r_{2}$ & $\Omega_{1} r_{1}^{2}$ & $\Omega_{2} r_{2}^{2}$ \\
\hline $\mathrm{a}$ & 0.0104 & 0.0092 & $1.76 \times 10^{-3}$ & $1.66 \times 10^{-3}$ \\
$\mathrm{~b}$ & 0.0139 & 0.0750 & $2.33 \times 10^{-3}$ & $9.04 \times 10^{-2}$ \\
$\mathrm{c}$ & 0.0081 & 0.0126 & $1.44 \times 10^{-3}$ & $4.83 \times 10^{-4}$ \\
$\mathrm{~d}$ & 0.0224 & 0.0154 & $1.25 \times 10^{-4}$ & $1.7 \times 10^{-3}$ \\
\hline
\end{tabular}

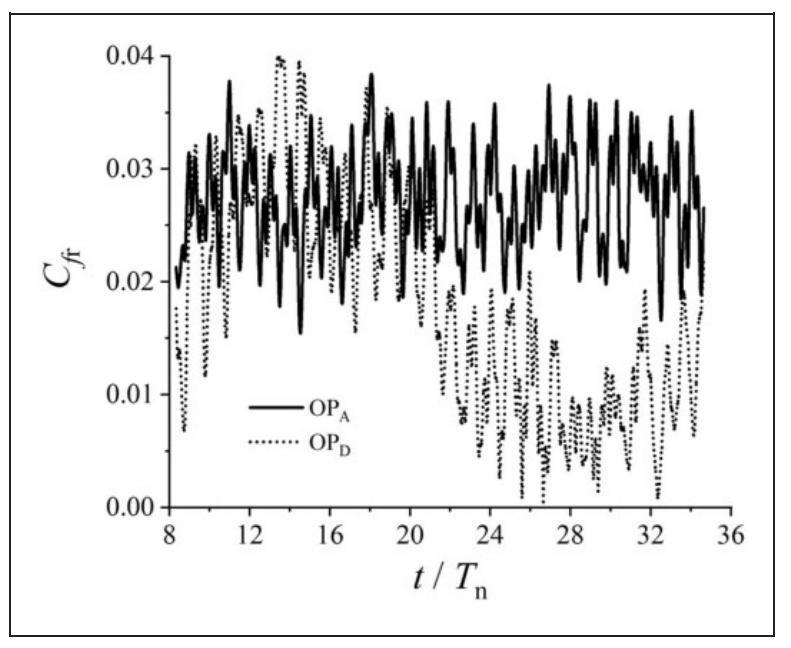

Figure 14. Unsteady radial force under two part-load conditions. (a)

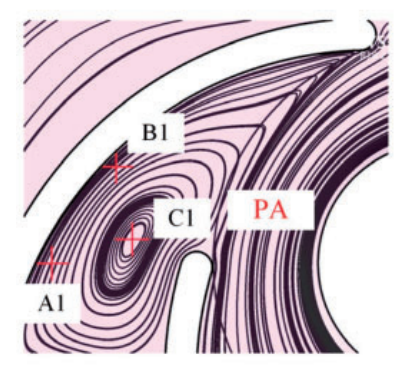

(b)

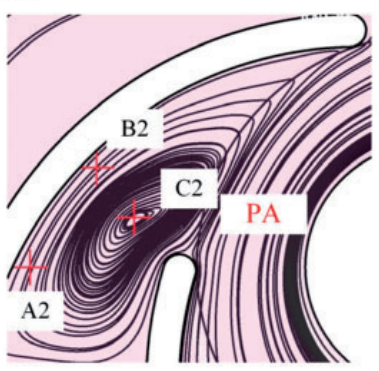

(c)

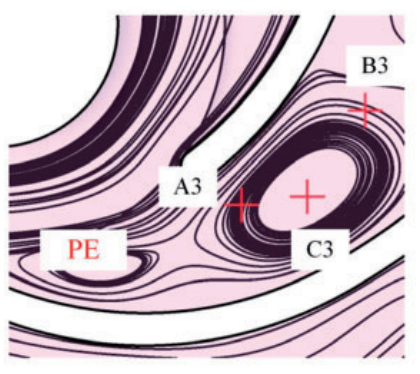

(d)

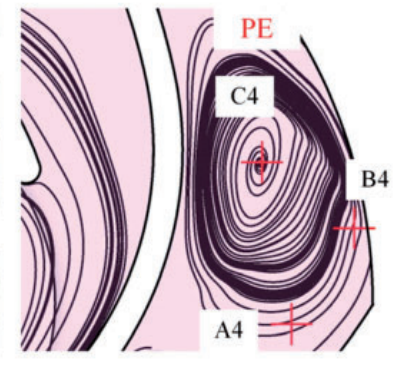

Figure 13. Vortices under the condition of: (a) $O P_{A}$ at $t=26 T_{n}$; (b) $O P_{A}$ at $t=30 T_{n}$; (c) $O P_{D}$ at $t=26 T_{n}$; (d) $O P_{D}$ at $t=30 T_{n}$. 
propagation of the rotating stall cells induces unsteady radial force, which is harmful to the units.

\section{Outlet pipe}

As stated by Newton et al., ${ }^{41}$ the source of entropy in an adiabatic device is related with the efficiency of fluid machinery. In the outlet pipe, the entropy generation rate per unit volume is defined by equation (24) to explore the dissipation of mechanical energy into entropy from the perspective of the viscous stress dissipation, which is one part of the head loss in the pump

$$
\sigma=\frac{1}{\bar{T}}\left(\overline{\tau_{\mathrm{ij}}} \frac{\partial \overline{u_{\mathrm{i}}}}{\partial x_{\mathrm{j}}}+\frac{\mu_{\mathrm{t}}}{\mu} \overline{\tau_{\mathrm{ij}}} \frac{\partial \overline{u_{\mathrm{i}}}}{\partial x_{\mathrm{j}}}\right)
$$

where $\bar{T}$ is the average temperature, and it is a constant in the current study, and $\bar{\tau}_{\mathrm{ij}}$ is a viscous stress tensor, defined by equation (25)

$$
\overline{\tau_{\mathrm{ij}}}=\lambda(\nabla \cdot u) \delta_{\mathrm{ij}}+2 \mu \varepsilon_{\mathrm{ij}}
$$

where $\lambda$ is the viscosity coefficient and $\varepsilon_{\mathrm{ij}}$ is the shear strain tensor.

As shown in Figure 15, significant entropy generation is mainly captured at the entrance of the outlet pipe of Pump $\mathrm{A}^{\prime}$ under condition of $\mathrm{OP}_{\mathrm{D}}$. The large value of entropy generation rate means large hydraulic loss due to the sudden change of flow direction there. The entropy generation rate decreases along the downstream direction in the outlet pipe.

\section{Head loss analysis based on energy equations}

As discussed above, the flow information under low flow rate conditions varies from that under positive slope conditions in Pump A'. In this section, head loss analysis based on energy equations is introduced to further explore the mechanism of positive slope under two typical conditions, $\mathrm{OP}_{\mathrm{A}}$ and $\mathrm{OP}_{\mathrm{D}}$, in Pump $\mathrm{A}^{\prime}$.

Wilhelm et al. ${ }^{42}$ established a detailed energy balance in a draft tube to investigate the head loss. Based on this concept, Lu et al. ${ }^{16}$ have developed the energy balance equations which are utilized for energy loss analysis in an airfoil, cascades, and a pump-turbine.

The energy balance equation is illustrated as follows

$$
\begin{aligned}
P_{\text {in }}= & P_{\mathrm{e}}+P_{1} \\
P_{1}= & -\rho g \Delta H Q_{v}=\iiint_{V} \frac{\partial}{\partial t}\left(\frac{1}{2} \rho \bar{u}^{2}\right) \mathrm{d} V \\
& -\iiint_{V} \frac{\partial\left(-\overline{u_{\mathrm{i}}} \overline{\rho u_{\mathrm{i}}^{\prime} u_{\mathrm{j}}^{\prime}}\right)}{\partial x_{\mathrm{j}}} \mathrm{d} V-\iiint_{V} \mu \frac{\partial\left(\overline{u_{\mathrm{i}}} \overline{D_{\mathrm{ij}}}\right)}{\partial x_{\mathrm{j}}} \mathrm{d} V \\
& +\iiint_{V}\left(-\overline{\rho u_{\mathrm{i}}^{\prime} u_{\mathrm{j}}^{\prime}}\right) \frac{\partial \overline{u_{\mathrm{i}}}}{\partial x_{\mathrm{j}}} \mathrm{d} V+\iiint_{V} \mu \overline{D_{\mathrm{ij}}} \frac{\partial \overline{u_{\mathrm{i}}}}{\partial x_{\mathrm{j}}} \mathrm{d} V \\
D_{\mathrm{ij}}= & \frac{\partial u_{\mathrm{i}}}{\partial x_{\mathrm{j}}}+\frac{\partial u_{\mathrm{j}}}{\partial x_{\mathrm{i}}} \\
-\overline{\rho u_{\mathrm{i}}^{\prime} u_{\mathrm{j}}^{\prime}}= & \mu_{\mathrm{t}}\left(\frac{\partial \overline{u_{\mathrm{j}}}}{\partial x_{\mathrm{i}}}+\frac{\partial \overline{u_{\mathrm{i}}}}{\partial x_{\mathrm{j}}}\right)-\frac{2}{3} \delta_{\mathrm{ij}} \rho k
\end{aligned}
$$

where $P_{\text {in }}$ is the input power, and $P_{\mathrm{e}}$ and $P_{1}$ stand for the effective power and power loss, respectively.

In equation (27), the first term on the right-hand side (named as $P_{11}$ ) is change rate of mean kinetic energy. The second term $\left(P_{12}\right)$ and third term $\left(P_{13}\right)$ are the diffusion of the Reynolds stress and viscous stress. The fourth term $\left(P_{14}\right)$ represents the turbulent kinetic energy production. And the last term $\left(P_{15}\right)$ is

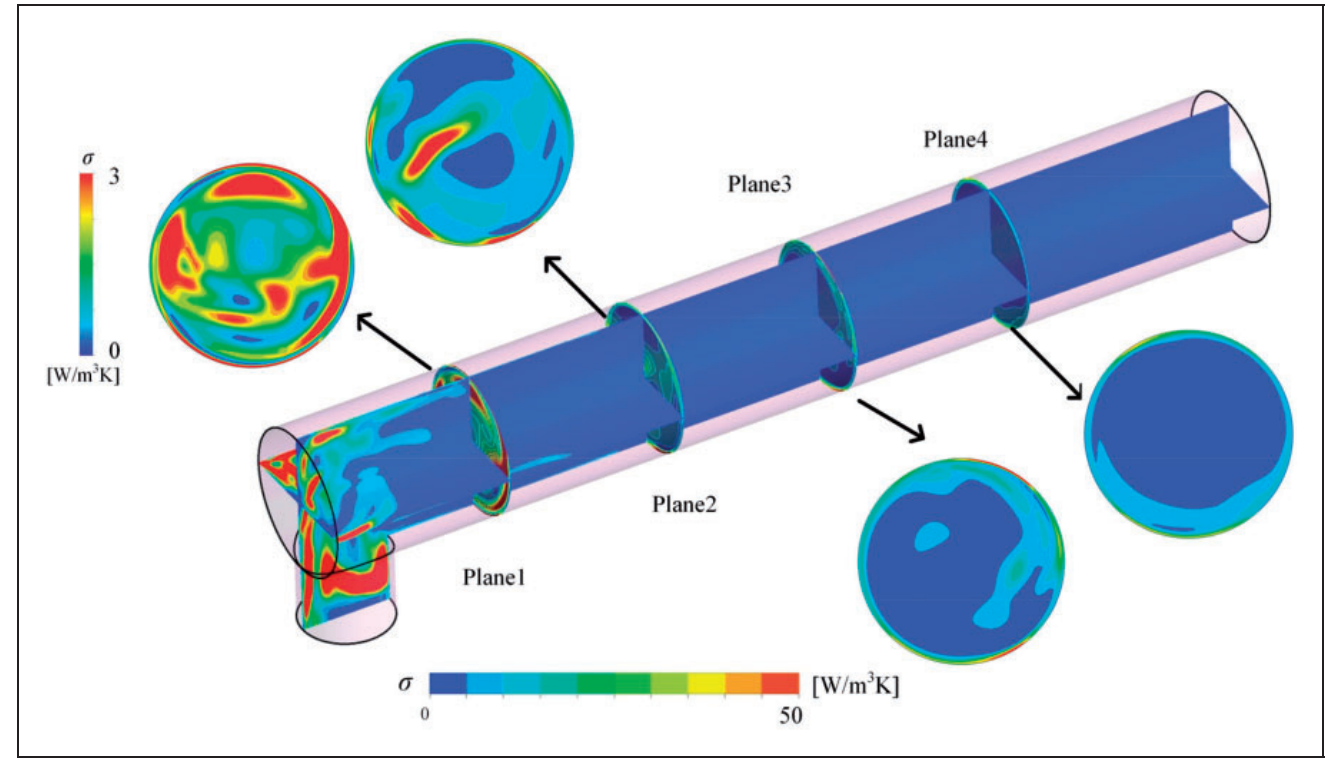

Figure 15. Entropy generation rate in outlet pipe of Pump $A^{\prime}$ under condition of $O P_{D}$. 
viscous dissipation of mean kinetic energy. As shown in equation (28), $D_{\mathrm{ij}}$ is a shear strain tenor. Based on Boussinesq approximation, Reynolds stress is organized according to equation (29). Other parameters: $\mu$ is dynamic viscosity, $\mu_{\mathrm{t}}$ is Eddy viscosity, and $\delta_{\mathrm{ij}}$ is Kronecker function.

Based on equation (27), equation (30) is applied to evaluate the head loss in a pump

$$
\Delta H=\frac{P_{1}}{-\rho g Q_{v}}
$$

The definition of head coefficient, i.e. equation (11) is used to achieve the non-dimensional head loss parameter $\Delta \psi$ for the head loss $\Delta H$. Consequently, the head loss components derived from equation (27) can be calculated. The non-dimensional parameters of head loss $\Delta \psi_{1} \sim \Delta \psi_{5}$ correspond to the power losses $P_{11} \sim P_{15}$.

In the high Reynolds number simulations, the term of diffusion of viscous stress $\left(\Delta \psi_{3}\right)$ is negligible in the current paper. In addition, compared with $\Delta \psi_{4}, \Delta \psi_{5}$, and $\Delta \psi_{2}, \Delta \psi_{1}$ (change rate of mean kinetic energy) is extremely small. Thus, Figure 16 shows the distributions of $\Delta \psi_{4}, \Delta \psi_{5}$, and $\Delta \psi_{2}$ in the impeller under four part-load conditions. It is noted that the term of turbulent kinetic energy production corresponding to $P_{14}$ or $\Delta \psi_{4}$ is the main component of head loss in the impeller of Pump $\mathrm{A}^{\prime}$, indicating that the mean kinetic energy is mainly transferred to turbulent kinetic energy. It is clear that head loss under the condition of $\mathrm{OP}_{\mathrm{C}}$ is the lowest. Compared with the condition of $\mathrm{OP}_{\mathrm{C}}$, the head loss under the condition of $\mathrm{OP}_{\mathrm{B}}$ is larger due to the strong separation flow in the impeller. It should be noted that the large head loss at $\mathrm{OP}_{\mathrm{B}}$ results in the positive slope in characteristic curve for Pump A'.

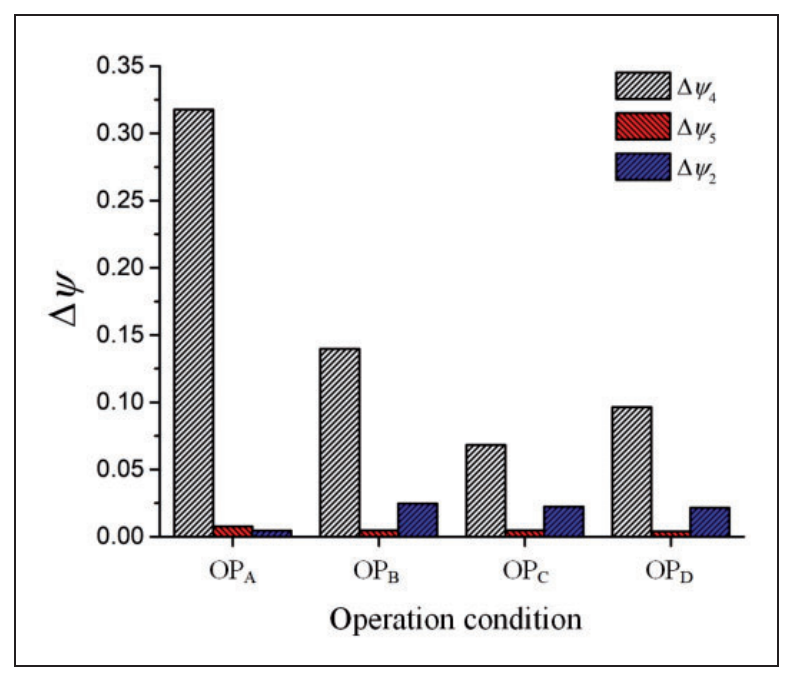

Figure 16. Head loss in the impeller of Pump $A^{\prime}$ under four part-load conditions.

\section{Conclusions}

In this study, a PANS model based on the standard $k-\varepsilon$ model is applied to investigate the unsteady flow in centrifugal pumps with different inlet pipe under part-load conditions. The flow instability is analyzed from different viewpoints such as Batchelor vortex family, R-T criterion, entropy generation rate, etc. Based on these results, the following can be concluded:

1. The present simulation using PANS model is acceptable compared with the experiment data for Pump A (i.e. the tested pump), and the current turbulence model is applicable to investigate the flow instability in a centrifugal pump.

2. No matter the geometry of the inlet pipe, the preswirling flows in the inlet pipe are in a CI region. Under the part-load condition of $\mathrm{OP}_{\mathrm{A}}$, the axial vorticity coefficient is affected by the geometries of inlet pipe. Under the part-load condition with rotating stall, e.g. $\mathrm{OP}_{\mathrm{D}}$, the flow in the inlet pipe is affected by the unstable flow in the pump impeller.

3. For the pump with straight inlet pipe, the vortex inside the blade-to-blade passages is in a stable state according to $\mathrm{R}-\mathrm{T}$ criterion under the condition of $\mathrm{OP}_{\mathrm{A}}$. And it is in the unstable state due to the centrifugal instability near the condition having positive slope (e.g. $\mathrm{OP}_{\mathrm{D}}$ ). The oscillations for pressure and radial force are dependent on the propagation of the rotating stall cells.

4. Head loss analysis based on energy equations elucidates that that turbulent kinetic energy production term is predominant in head loss in pump impeller.

\section{Declaration of Conflicting Interests}

The author(s) declared no potential conflicts of interest with respect to the research, authorship, and/or publication of this article.

\section{Funding}

The author(s) disclosed receipt of the following financial support for the research, authorship, and/or publication of this article: National Key R\&D Program of China (2018YFB0606101), the National Natural Science Foundation of China (No. 51536008), Beijing Natural Science Foundation (3182014) and the Tsinghua National Laboratory for Information Science and Technology.

\section{ORCID iDs}

Weixiang Ye (D) https://orcid.org/0000-0002-6740-3192 Zhongdong Qian (D) https://orcid.org/0000-0001-9409-9838 Xiaojun Li (D) https://orcid.org/0000-0002-5530-1666 Zuchao Zhu (D) https://orcid.org/0000-0003-4170-8374

\section{References}

1. Chanphavong L, Al-Attab KA and Zainal ZA. Swirl flow field analysis of producer gas premixed charge in a flameless cyclone combustor. Int J Energy Res 2018; 42: 4360-4371. 
2. Susan-Resiga R, Ciocan GD, Anton I, et al. Analysis of the swirling flow downstream a Francis turbine runner. J Fluid Eng 2006; 128: 177-189.

3. Zhang RK, Mao F, Wu JZ, et al. Characteristics and control of the draft-tube flow in part-load Francis turbine. J Fluids Eng 2009; 131: 021101.

4. Yang DD, Luo XW, Liu DM, et al. Unstable flow characteristics in a pump-turbine simulated by a modified partially-averaged Navier-Stokes method. Sci Chin Technol Sci 2019; 62: 406-416.

5. Widmer C, Staubli $\mathrm{T}$ and Ledergerber N. Unstable characteristics and rotating stall in turbine brake operation of pump-turbines. J Fluid Eng 2011; 133: 041101.

6. Ye WX, Huang RF, Jiang ZW, et al. Instability analysis under part-load conditions in centrifugal pump. $J$ Mech Sci Technol 2019; 33: 269-278.

7. Veres JP. A survey of instability within centrifugal pumps and concepts for improving the flow range of pumps in rocket engines. In: JANNAF propulsion meeting, Indianapolis, IN, USA, 1992.

8. Rayleigh L. On the dynamics of revolving fluids. Proc $R$ Soc Lond Ser A: Contain Pap Math Phys Charact 1917; 93: $148-154$.

9. Hoffmann ER and Joubert PN. Turbulent line vortices. J Fluid Mech 1963; 16: 395-411.

10. Batchelor GK. Axial flow in trailing line vortices. J Fluid Mech 1964; 20: 645-658.

11. Lessen M, Singh PJ and Paillet F. The stability of a trailing line vortex, part 1: inviscid theory. J Fluid Mech 1974; 63: 753-763.

12. Delbende I, Chomaz JM and Hurre P. Absolute/convective instabilities in the Batchelor vortex a numerical study of the linear impulse response. J Fluid Mech 1998; 355: 229-254.

13. Galván S, Reggio M and Guibault F. Numerical optimization of the inlet velocity profile ingested by the conical draft tube of a hydraulic turbine. J Fluid Eng 2015; 137: 071102.

14. Li XJ, Gao PL, Zhu ZC, et al. Effect of the blade loading distribution on hydrodynamic performance of a centrifugal pump with cylindrical blades. $J$ Mech Sci Technol 2018; 32: 1161-1170.

15. Fu XL, Li DY, Wang HJ, et al. Dynamic instability of a pump-turbine in load rejection transient process. Sci Chin Technol Sci 2018; 61: 1765-1775.

16. Lu GC, Zuo ZG, Liu DM, et al. Energy balance and local unsteady loss analysis of flows in a low specific speed model pump-turbine in the positive slope region on the pump performance curve. Energies 2019; 12: 1829.

17. Yan P, Chu N, Wu DZ, et al. Computational fluid dynamics-based pump redesign to improve efficiency and decrease unsteady radial forces. J Fluid Eng 2017; 139: 011101.

18. Dou HS and Jiang W. Application of energy gradient theory in flow instability in a centrifugal pump. IOP Conf Ser Mater Sci Eng 2013; 52: 012007.

19. Wo AM and Bons JP. Flow physics leading to system instability in a centrifugal pump. J Turbomach 1994; 116: 612-620.

20. Krause N, Zähringer K and Pap E. Time-resolved particle imaging velocimetry for the investigation of rotating stall in a radial pump. Exp Fluid 2005; 39: 192-201.
21. Liu DM. Numerical analysis of the channel vortices and vortices in draft tube of a Francis turbine based on the cavitation calculation. $\mathrm{PhD}$ thesis, China: Tsinghua University, 2011.

22. Ji B, Luo XW, Arndt REA, et al. Numerical simulation of three dimensional cavitation shedding dynamics with special emphasis on cavitation-vortex interaction. Ocean Eng 2014; 87: 64-77.

23. Luo DH, Yan C, Liu HK, et al. Comparative assessment of PANS and DES for simulation of flow past a circular cylinder. $J$ Wind Eng Ind Aerodyn 2014; 134 : 65-77.

24. Huang RF, Luo XW and Ji B. Numerical simulation of the transient cavitating turbulent flows around the Clark-Y hydrofoil using modified partially averaged Navier-Stokes method. J Mech Sci Technol 2017; 31: 2849-2859.

25. Fadai-Ghotbi A, Friess C, Manceau R, et al. A seamless hybrid RANS-LES model based on transport equations for the subgrid stresses and elliptic blending. Phys Fluid 2010; 22: 055104.

26. Xia JL, Yadigaroglu G, Liu YS, et al. Numerical and experimental study of swirling flow in a model combustor. Int J Heat Mass Transf 1998; 41: 1485-1497.

27. Yaras MI and Grosvenor AD. Evaluation of one- and two-equation low-Re turbulence models, part I: axisymmetric separating and swirling flows. Int $J$ Numer Methods Fluid 2003; 42: 1293-1319.

28. Gnanaskandan A and Mahesh K. Large eddy simulation of the transition from sheet to cloud cavitation over a wedge. Int J Multiph Flow 2016; 83: 86-102.

29. Foroutan H and Yavuzkurt SA. Partially-averaged Navier-Stokes model for the simulation of turbulent swirling flow with vortex breakdown. Int $J$ Heat Fluid Flow 2014; 50: 402-416.

30. Huang RF, Luo XW, Ji B, et al. Turbulent flows over a backward facing step simulated using a modified partially averaged Navier-Stokes model. J Fluid Eng 2017; 139: 044501 .

31. Girimaji SS. Partially-averaged Navier-Stokes model for turbulence: a Reynolds-averaged Navier-Stokes to direct numerical simulation bridging method. $J$ Appl Mech-Trans ASME 2006; 73: 413-421.

32. Lakshmipathy S and Girimaji SS. Partially averaged Navier-Stokes (PANS) method for turbulence simulations: flow past a circular cylinder. J Fluid Eng 2010; 132: 121202 .

33. Liu JT, Guo PC, Chen TJ, et al. The influence of nonlinear shear stress on partially averaged Navier-Stokes (PANS) method. J Hydrodyn 2017; 29: 479-484.

34. Wilcox DC. Reassessment of the scale-determining equation for advanced turbulence models. AIAA $J$ 1988; 26: 1299-1310.

35. Lakshmipathy S and Girimaji SS. Partially-averaged Navier-Stokes method for turbulent flows: $k-\omega$ model implementation. In: Collection of technical papers - 44th AIAA aerospace sciences meeting and exihibit, Reno, Nevada, 9-12 January 2006, pp.15041518. United States: American Institute of Aeronautics and Astronautics (AIAA).

36. Luo DH, Yan C and Wang XY. Computational study of supersonic turbulent-separated flows using partially averaged Navier-Stokes method. Acta Astronaut 2015; 107: 234-246. 
37. Menter FR. Two-equation Eddy-viscosity turbulence models for engineering applications. AIAA J 1994; 32: 1598-1605.

38. Girimaji SS and Abdol-Hamid KS. Partially-averaged Navier Stokes model for turbulence: implementation and validation. In: 43rd AIAA aerospace sciences meeting and exhibit, Reno, NV, USA, 10-13 January 2005.

39. Launder BE and Spalding DB. Lectures in mathematical models of turbulence. Cambridge, MA: Academic Press, 1972.

40. Zhang RK, Cai QD and Wu JZ. The physical origin of severe low-frequency pressure fluctuations in giant Francis turbines. Mod Phys Lett B 2005; 19: 1527-1530.

41. Newton P, Copeland C, Martinez-Botas R, et al. An audit of aerodynamic loss in a double entry turbine under full and partial admission. Int $J$ Heat Fluid Flow 2012; 33: 70-80.

42. Wilhelm S, Balarac G, Metais O, et al. Analysis of head losses in a turbine draft tube by means of 3D unsteady simulations. Flow Turbul Combust 2016; 97: 1255-1280

\section{Appendix}

\section{Notation}

$a$

external axial velocity of a Batchelor vortex

$C_{\text {fri }}$

$C_{\mathrm{p}}$

$C P_{\text {sd }}$

$D^{*}$

$f_{\mathrm{k}}$

$f_{\varepsilon}$

$k$

$p_{\mathrm{i}}^{*}$

amplitude

standard deviation of pressure

fluctuation

diameter of inlet tube

unresolved-to-total ratio of turbulent

kinetic energy

unresolved-to-total ratio of dissipation

turbulent kinetic energy

instantaneous pressure
$P$

$P_{\mathrm{e}}$

$P_{\text {in }}$

$P_{1}$

$q$

$Q^{*}$

$\left(r^{*}, \theta^{*}, z^{*}\right)$

$R^{*}$

$\mathrm{u}$

$U^{*}\left(r^{*}\right)$

$v_{\mathrm{i}}$

$V^{*}\left(r^{*}\right)$

$V_{\mathrm{i}}$

$V_{\mathrm{i}}^{*}$

$V_{0}^{*}$

$W^{*}\left(r^{*}\right)$

$W_{\mathrm{c}}^{*}$

$W_{\infty}^{*}$

$\varepsilon$

$\phi$

$\eta$

$\tau$

$\tau\left(V_{\mathrm{i}}^{*}, V_{\mathrm{j}}^{*}\right)$

$v$

$\omega_{\mathrm{z}}$ production term of $k$

effective power

input power

power losses

swirl ratio of a Batchelor vortex

volume flow rate at inlet tube

radial, azimuthal, and axial coordinates

of the cylindrical system

a measure of the vortex core radius of a

Batchelor vortex

a superscript denoting unresolved term radial velocity

fluctuation component of velocity

azimuthal velocity

partially averaged velocity $V_{\mathrm{i}}$

instantaneous velocity

averaged velocity at the inlet tube

axial velocity

centerline axial velocity of a Batchelor vortex

freestream axial velocity of a Batchelor vortex

turbulent dissipation rate

flow rate coefficient

efficiency

power coefficient

subfilter scale tensor

Eddy viscosity

axial vorticity coefficient of a Batchelor vortex

entropy generation rate per unit volume head coefficient

the local grid size

Taylor turbulence length scale

rotation rate at the axis of a Batchelor

vortex 